\title{
A Wavelet Modulation Theorem for Bandlimited Signals
}

\author{
G.A.A. Araújo, H.M. de Oliveira
}

\begin{abstract}
This paper presents a variant of the classical modulation theorem by considering continuous wavelets instead of (perpetual) sinusoidal signals from an oscillator. The bandwidth requirements for transmission are evaluated for several continuous wavelets, including Shannon, Mexican hat, Haar, gauss1, Meyer, de Oliveira, Morlet, and beta wavelets.

Resumo - Este artigo apresenta uma variante do clássico teorema da modulação, considerando wavelets continuas ao invés de senoidais perpetuas de um oscilador. Os requisitos de banda passante para transmissão são avaliados para diversas wavelets continuas, incluindo aquelas de Shannon, sombrero, Haar, gauss1, Meyer, de Oliveira, Morlet, e wavelets beta.
\end{abstract}

Index Terms - modulation theorem, wavelets, bandwidth.

\section{INTRODUCTION}

$\mathrm{W}$ AVELET has long been used as a powerful and deeprooted tool in signal analysis with applications in several different fields [1-5]. An extensive list of applications in Telecommunications can be found in $[6$, Chap.6, p.197]. Three among them have been suggested in former SBrT/ITS, namely, a spread-spectrum system based on finite field wavelets [7], a digital wavelet-based modulation (wavelet-shift keying) [8], a multiresolution division multiplex [9].

The (AM) modulation theorem is one of the most celebrated and widely applied results of the Communication Theory [10]. By the same token as standard analog modulations, two kinds of "wavelet modulation" between a signal $f(t)$ and a continuous wavelet $\psi_{\mathrm{a}, \mathrm{b}}(t)$ used as a (short pulses) carrier can be devised:

$$
\begin{array}{ll}
\text { 1. AM-type } & f(t) \cdot \psi_{a, b}(t) \\
\text { 2. FM-type } & \psi_{a_{0}+K f(t), b}(t)
\end{array}
$$

Figure 1 shows a couple of examples of a single tone wavelet-modulated with a wavelet.

In this paper we are concerned with the first case: What is the spectrum of the wavelet-modulated signal? Which are the bandwidth requirements in this case? These two naïve questions are answered in the sequel.

This work was partially supported by the Brazilian National Council for Scientific and Technological Development $(\mathrm{CNPq})$ under research grant \#306180 (HMdO). GAAA is grateful to CNPq for support. The authors are with the Signal Processing Group, Federal University of Pernambuco, C.P. 7800, CEP: 50711-970, Recife-PE, Brazil

(e-mail: giovanna.angelis@gmail.com hmo@ufpe.br)
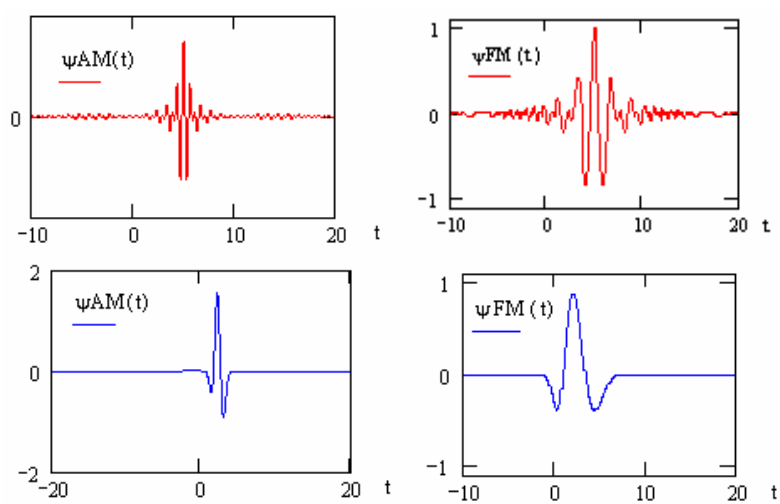

Figure 1. A single tone of $50 \mathrm{kHz}$ wavelet-modulated with a wavelet pulses (time scale in $\mu \mathrm{s}$ ): (a) Shannon(sinc)-wavelet AM-type, scale $a=0.5 \mu$ translation $b=5 \mu$ and $\mathrm{f}_{0}=1.414 \mathrm{MHz}$; (b) Shannon(sinc)-wavelet FM-type, scale $a=1 \mu$ translation $b=5 \mu, \mathrm{K}=0.25$. (c) Mexican hat wavelet AM-type, scale $a=0.5 \mu$, translation $b=2 \mu$; (d) Mexican hat wavelet FM-type, scale $a=1 \mu$ translation $b=2 \mu, \mathrm{K}=0.25$.

The paper is organized as follows. Section 2 presents a brief review on continuous wavelets, collecting analytical expressions in frequency domain, which are required to evaluate the bandwidth requirements of wavelet-modulated signals. The next section discusses about time and band limited feature of signals, assessing $2 B T$-theorem as well as Gabor resolution. Section 4 addresses the AM-wavelet modulation, computing relevant parameter of wavelets and bandwidth requirements of the modulation, and some final remarks are presented in Section 5.

\section{REVIEWING CONTINUOUS WAVELETS}

Despite the fact that analytical expressions for the spectra of continuous wavelets are known [12], they are collected in Table I for a number of selected wavelets of interest. The corresponding waveforms in both time and frequency domains are exhibitd in the Appendix B. Both infinite supported wavelets (Shannon, Morlet, Mexhat, Meyer, de Oliveira) and compactly supported wavelets (Haar, hat, beta) were considered. In that way, a broad variety of spectral shapes was taken into account. Most of these wavelets can be found in [12], [13], but [14], [15]. 
Table I. Analytical expression of known continuous wavelets whose parameters concerning modulation were computed.

\begin{tabular}{|c|c|}
\hline $\begin{array}{l}\text { Mother } \\
\text { wavelet }\end{array}$ & $\begin{array}{l}\text { Frequency } \\
\text { Spectrum }\end{array}$ \\
\hline Shannon & $\mid \begin{array}{lc}1 & \text { if } \\
0 & \text { otherwise }\end{array}$ \\
\hline Morlet & $\sqrt{2} \frac{1}{\sqrt[4]{\pi}} e^{-\left(w-w_{0}\right)^{2} / 2}$ \\
\hline $\begin{array}{l}\text { Mexican } \\
\text { hat }\end{array}$ & $\sqrt{8} \frac{\sqrt[4]{\pi}}{\sqrt{3}} \cdot w^{2} \cdot e^{-w^{2} / 2}$ \\
\hline Haar & $\begin{array}{c}j \sqrt{2} \cdot \operatorname{Sa}(w / 2) \cdot \sin (w / 2) \\
\operatorname{Sa}(x):=\sin (x) / x\end{array}$ \\
\hline gauss 1 & $-2 j \cdot \sqrt[4]{\pi} \cdot w \cdot e^{-w^{2} / 2}$ \\
\hline Meyer & $\Psi$ mey $(w):=\mid \begin{array}{l}1 \cdot \sin \left(\frac{\pi}{2} \cdot v\left(\frac{3}{2 \cdot \pi} \cdot|w|-1\right)\right) \cdot \mathrm{e}^{-\sqrt{-1} \cdot \frac{\mathrm{w}}{2}} \text { if } \frac{2 \cdot \pi}{3} \leq|w| \leq \frac{4 \pi}{3} \\
1 \cdot \cos \left(\frac{\pi}{2} \cdot v\left(\frac{3}{4 \pi} \cdot|w|-1\right)\right) \cdot \mathrm{e}^{-\sqrt{-1} \cdot \frac{\mathrm{w}}{2}} \text { if } \frac{4 \pi}{3} \leq|\omega| \leq \frac{8 \cdot \pi}{3} \\
0 \text { otherwise }\end{array}$ \\
\hline $\mathrm{deO}$ & $\begin{array}{l}\Psi^{(d e O)}(w)=e^{-j w / 2} S^{(d e O)}(w), \\
S^{(d e O)}(w)= \begin{cases}0 & \text { se } w<\pi(1-\alpha) \\
\frac{1}{\sqrt{2 \pi}} \cos \frac{1}{4 \alpha}(w-\pi(1+\alpha)) & \text { se } \pi(1-\alpha)<w<\pi(1+\alpha) \\
\frac{1}{\sqrt{2 \pi}} & \text { se } \pi(1+\alpha)<w<2 \pi(1-\alpha) \\
\frac{1}{\sqrt{2 \pi}} \cos \frac{1}{8 \alpha}(w-2 \pi(1-\alpha)) & \text { se } 2 \pi(1-\alpha)<w<2 \pi(1+\alpha) \\
0 & \text { se } w>2 \pi(1+\alpha)\end{cases} \end{array}$ \\
\hline beta & $\begin{array}{l}\frac{w}{T(\alpha, \beta)} \cdot \operatorname{MKUMMER}(\alpha, \beta, w T(\alpha, \beta)) \\
\text { where } \mathrm{T}(\alpha, \beta):=(\alpha+\beta) \cdot \sqrt{\frac{(\alpha+\beta+1)}{\alpha \cdot \beta}}\end{array}$ \\
\hline
\end{tabular}

\section{TIMELIMITED VERSUS BANDLIMITED SIGNALS}

It is well known that a signal cannot be simultaneously limited in time and frequency [16, p.352]. However, it is quite helpful to assume some approximations such as these described in [17]. Many other approaches has been used to handle this situation, offering useful approximations such as the one described by the 2BT-theorem by Landau and Pollack [18]. Let $f(t) \leftrightarrow F(w)$ be a real signal with finite energy, $E=\int_{-\infty}^{+\infty} f^{2}(t) d t$, time-limited in $T$ seconds, whose energy content outside a given band $B(\mathrm{~Hz})$ is less that $\lambda E(\lambda$ is the fraction of energy outside the considered band).Then:

$$
\begin{aligned}
& >f(t)=0,|t|>T / 2 \\
& >\quad \int_{-B}^{+B}|F(w)|^{2} d f>(1-\lambda) E .
\end{aligned}
$$

Theorem 2BT [LAN\&POLL 1961]. There exists a ensemble of orthogonal signals $\left\{\phi_{m}(t)\right\}_{m=0}^{L-1}$, with finite cardinality $L$, such that the energy of the error signal from the approximation of a truncated series is less that $12 \lambda E$, i.e.,

$$
f(t) \cong \sum_{m=0}^{L-1} c_{m} \phi_{m}(t)
$$

such that $\quad \int_{-\infty}^{+\infty}\left|f(t)-\sum_{m=0}^{L-1} c_{m} \phi_{m}(t)\right|^{2} d t<12 \lambda E, \quad$ where $L=\lfloor 2 B T+1\rfloor$.
Therefore, the number of dimensions required to represent a signal almost limited in both time and frequency (duration $T$ and bandwidth $B$ ) is so that $T . B \geq 0.5$.

Consequently, as a practical model, it is assumed a bandlimited signal $f(t)$ with a maximum frequency $B$ and an effective duration $T$, or a timelimited signal $f(t)$ with a effective duration bandwidth $B$.

Another especially powerful approach to model a signal as approximately simultaneously finite in both domains is to consider the rms-bandwidth and the rms-duration, as proposed by Gabor to study time-frequency resolution. Gabor [19] derived a relationship similar to the uncertainty principle of Heisenberg. Let $f(t)$ be a signal of finite energy, and $F(w)$ its transform, thus proving that the spreading in time and frequency cannot be assigned in an uncoupled way. The moments of the signal are defined by:

$$
\overline{t^{n}}:=\frac{\int_{-\infty}^{+\infty} f^{*}(\varsigma) \varsigma^{n} f(\varsigma) d \varsigma}{\int_{-\infty}^{+\infty} f^{*}(\varsigma) f(\varsigma) d \varsigma}, \overline{w^{n}}:=\frac{\int_{-\infty}^{+\infty} F^{*}(\varsigma) \varsigma^{n} F(\varsigma) d \varsigma}{\int_{-\infty}^{+\infty} F^{*}(\varsigma) F(\varsigma) d \varsigma} .
$$

The effective duration (effective bandwidth, respectively) of a signal $f(t)$ (respectively $F(w))$ can be defined via:

$$
\begin{aligned}
& \Delta_{t}:=\sqrt{\overline{(t-\bar{t})^{2}}} \quad \text { r.m.s. duration, } \\
& \Delta_{w}:=\sqrt{\overline{(w-\bar{w})^{2}}} \text { r.m.s. bandwidth, }
\end{aligned}
$$

$\Delta_{t}$ and $\Delta_{w}$ correspond to the standard deviations, classical measures of spreading (the measures here are a bit different than those in the original paper). Following arguments from the quantum mechanics, he found: $\Delta_{t} . \Delta_{w} \geq 1 / 2$.

De Oliveira [20], who derived a lower bound on the product of the time-entropy and frequency-entropy of wavelets obtained another rather similar uncertainty principle.

Proposition $1[6]$. Any real signal $f(t) \leftrightarrow F(w)$ such that $f, f$, $F, F^{\prime} \in L^{2}(\mathbb{R})$, has finite resolutions given by

$$
\Delta_{t}^{2}=\frac{\int_{-\infty}^{+\infty}\left|F^{\prime}(w)\right|^{2} d w}{\int_{-\infty}^{+\infty}|F(w)|^{2} d w}<+\infty, \quad \Delta_{w}{ }^{2}=\frac{\int_{-\infty}^{+\infty}\left|f^{\prime}(t)\right|^{2} d t}{\int_{-\infty}^{+\infty}|f(t)|^{2} d t}<+\infty
$$

Particularly, the resolutions of beta wavelets of parameters $\alpha$ and $\beta$ [15] were investigated, and close expression derived (shown in Appendix A).

Two (approximately and simultaneously) limitation are assumed for a signal $f(t)$, namely $B$ as bandwidth and $T$ as duration.

If the bandwidth $B$ of a signal is estimated by a suitable criterion (say first zero of its spectrum, Gabor rmsbandwidth, fractional content of energy etc.) then its duration $T$ can be assumed inversely proportional to the band:

$$
\left\{\begin{array}{cc}
T . B \geq 0.5 \quad(2 \mathrm{BT}-\text { theorem) } \\
\Delta_{t} \cdot \Delta_{w} \geq 0.5 \quad \text { (Gabor }- \text { Heisenberg inequality) }
\end{array}\right.
$$

Proposition 2 (time-frequency wavelet confinement). A direct application of these relationships furnishes a bound on the supports of a continuous wavelet $\psi(t) \leftrightarrow \Psi(w)$ :

effSup $\psi$. effSup $\Psi \geq 0.5$, here effSup denotes the length of the effective support of the signal.

Proof. Directly from Gabor-Heisenberg/2BT-theorem. 


\section{ON THE WAVELET MODULATION}

Two parameters are defined for the modulation signal and the wavelet pulse:

$T_{m}:=\sup _{t \in R}\{|t|$ such that $|f(t)| \neq 0\}$ and

$T_{\psi}:=\sup _{t \in R}\{|t|$ such that $|\psi(t)| \neq 0\}$ for timelimited signals.

If the signals are not compactly supported, then the definition of the effective support is considered. If the signal is centered at the origin (i.e. $\bar{t}=0$ ), then $T_{m}=T / 2$ and for compactly supported wavelets, $T_{\psi}=\operatorname{effsup} \psi / 2$.

Table II. Parameter of known continuous wavelets: wavelet duration (rms, and time effective support), wavelet bandwidth ( $\mathrm{rms}$ and frequency effective support).

\begin{tabular}{lcccc}
\hline \multicolumn{1}{c}{$\begin{array}{c}\text { Mother } \\
\text { wavelet }\end{array}$} & $\Delta_{t}$ & $\begin{array}{c}\text { Duration } T \\
\text { length } \\
\text { effSup } \Psi\end{array}$ & $\Delta_{f}=\Delta_{w} / \sqrt{2 \pi}$ & $\begin{array}{c}\text { length } \\
\text { effSup } \Psi\end{array}$ \\
\hline Shannon & 2.023 & 40.000 & 1.915 & 0.500 \\
Morlet & 0.707 & 3.642 & 0.273 & 0.739 \\
Mex hat & 1.080 & 5.376 & 0.631 & 0.874 \\
Haar & 0.577 & 2.000 & 1.039 & 31.381 \\
Gauss1 & 1.225 & 4.764 & 0.489 & 0.758 \\
Meyer & 0.477 & 3.678 & 1.960 & 1.000 \\
deO0.33 & 0.477 & 3.700 & 0.461 & 1.000 \\
beta(3,4) & 1.445 & 5.715 & 2.895 & 5.952 \\
\hline
\end{tabular}

Resolutions $\Delta_{t}$ and $\Delta_{f}=\Delta_{w} / \sqrt{2 \pi}$ were computed by either definition or Proposition 1. The length effsup was computed on the basis of $99 \%$ fractional energy content (Table 1).

$$
\begin{array}{r}
\int_{-t_{99}}^{t_{99}} \psi^{2}(t) d t=0.99, \text { length effsup } \psi=2 . t_{99}, \\
\frac{1}{2 \pi} \cdot \int_{-w_{99}}^{w_{99}}|\Psi(w)|^{2} d w=0.99, \text { length effsup } \Psi=\frac{w_{99}}{\pi} .
\end{array}
$$

The duration and bandwidth (wavelet length) was given by the length $T_{\psi}$ and $B_{\Psi}$ for compactly supported in time (e.g. Haar, beta) or frequency (e.g. Shan, deO, Meyer), respectively. The bandwidth requirements of wavelet modulation for transmission are evaluated for a number of continuous wavelets as shown in Table II. As expected, the wavelet scale plays a role similar to the one of the carrier frequency.

Table III. Parameter of known continuous wavelets: frequency band of confinement, wavelet nominal central frequency of the mother wavelet.

\begin{tabular}{llc}
\hline $\begin{array}{c}\text { Mother } \\
\text { wavelet }\end{array}$ & $\begin{array}{c}\text { Frequency band } \\
{[L, U]}\end{array}$ & $\begin{array}{c}\text { wavelet nominal carrier } \\
\text { frequency } f_{o}\end{array}$ \\
\hline Shannon & {$[0.5,1.0]$} & $\sqrt{2} / 2 \cong 0.707$ \\
Morlet & {$[0.136,0.819]$} & 0.334 \\
Mex hat & {$[0.044,0.498]$} & 0.148 \\
Haar & {$[0.022,0.858]$} & 0.137 \\
Gauss1 & {$[0.01,0.440]$} & 0.066 \\
Meyer & {$[0.333,1.333]$} & $2 / 3 \cong 0.667$ \\
deO & {$[0.333,1.333]$} & $2 / 3 \cong 0.667$ \\
beta & {$[0.001,0.512]$} & 0.072 \\
\hline
\end{tabular}

The borders of the frequency band, $L$ and $U$, were derived by considering below $10 \%$ of the maximum value of $|\Psi(w)|$ in a criterion inspired in the Carson rule for FM bandwidth evaluation [10]. The nominal carrier frequency is estimated via the geometric mean between $L$ and $U$.

Clearly $f(t) \cdot \psi_{a, b}(t)=0$ if $|b|>\frac{T+T_{\psi} / a}{2}$ so the modulated signal has a term $u\left(\frac{T+T_{\psi} / a}{2}-|b|\right)$.

Which is the top wavelet choice for modulation? It can be seen that all listed wavelets in Table II have roughly the same support in the frequency domains, excluding Haar wavelet, due to its discontinuous feature. Shannon wavelet is not a good candidate due to its high time duration. deO0.33 was conceived using Nyquist filters [10] and seems to provide a reasonable choice.

Proposition 3. The wavelet modulation of a signal $f(t)$ by a daughter wavelet is (essentially) bounded in the range given by: $f(t) \cdot \psi_{a, b}(t)=S_{a, b}(t) \cdot u\left(\frac{T+T_{\psi} / a}{2}-|b|\right)$.

Corollary 1. The continuous wavelet transform is limited in scale and according with:

$$
C W T(a, b)=S(a, b) \cdot u\left(\frac{T+T_{\psi} / a}{2}-|b|\right) \text {. }
$$

Considering a wavelet $\psi(t) \leftrightarrow \Psi(w)$ with effective support in the frequency domain given by $\operatorname{effSup} \Psi(w)$, then the CWT is (essentially) constrained within the range

$$
u\left(\frac{T}{2}+\frac{\operatorname{effSup} \psi / 2}{a}-|b|\right) .
$$

The convolution theorem [10] is used to evaluate the wavelet-modulated spectrum.

Proposition 4 (bandwidth requirements of a wavelet modulation). For a scale $a \neq 0$, the product $f(t) \cdot \psi_{a, b}(t)$ demands an effective bandwidth $W \cong 2 B+\frac{\operatorname{effSup} \Psi(w)}{a}$, which is greater than the amplitude modulation (AM) requirements, as expected.

Proof. The spectrum of the wavelet modulated signal is

$$
\mathfrak{I C W T}\left\{f(t) \cdot \psi_{a, b}(t)\right\}=S(w) \cdot \prod\left(\frac{w-\frac{U+L}{2 a}}{\frac{U-L}{a}+2 B}\right)
$$

with $|a|<L / B$, where $L$ and $U$ are the borders of the mother wavelet bandpass spectrum.

Let $f_{0}$ be the (normalized) central frequency of the wavelet.

$$
f(t) \cdot \psi_{a, b}(t) \leftrightarrow S(f) \cdot \prod\left(\frac{f-\frac{f_{0}}{a}}{\frac{e f f S u p \Psi}{a}+2 B}\right) u\left[\left(\frac{T}{2}-\frac{\text { effSup } \psi}{2 a}-|b|\right)\right]
$$

then the proof follows by evaluating the length of gate pulse.

It can be remarked that when the wavelets becomes arbitrarily large $\lim \psi_{a, b}(t)$, the "wavelet" has it support $a \rightarrow 0$

reduced to a punctual support [see 21] and $W \cong 2 B$, as expected, i.e., twice the signal bandwidth. It is also worthwhile to remark that the greatest frequency of the bandlimited signal is assumed to be less than the lower limit 
of the effective frequency range of the wavelet spectrum. In this way the wavelet can be taken as a short carrier pulse, otherwise the high frequency signal will play the role of carrier as illustrated in Figure 2.
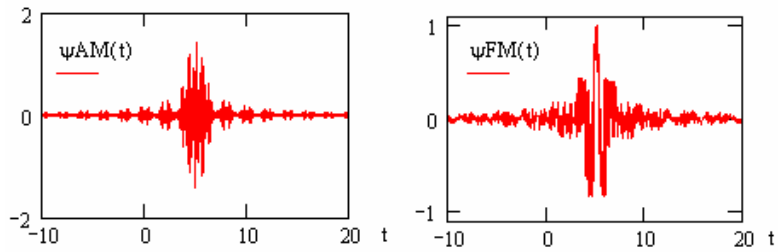

Figure 2. A single tone of $10 \mathrm{MHz}$ modulates a wavelet pulses ( $t$ in $\mu$ s): (a) Shannon(sinc)-wavelet AM-type, scale $a=1 \mu$ translation $b=5 \mu$ and $f_{0}=707$ $\mathrm{kHz}$; (b) Shannon(sinc)-wavelet FM-type, scale $a=1 \mu$ translation $b=5 \mu$, $\mathrm{K}=0.25$.

Inspired in the (universal) synchronous detection of AM [10], we evaluate the demodulator assuming a product by the same wavelet carrier, yielding $f(t) \cdot \psi_{a, b}^{2}(t)$. The lowpass component of this signal is expected to contain the original signal. Examining a few particular cases, it was found that the square root of the $\psi_{a, b}^{2}(t)$ yielded envelope waveforms rather similar with those of the full-rectified scale function. This is illustrated in Fig. 3 for a few wavelets.
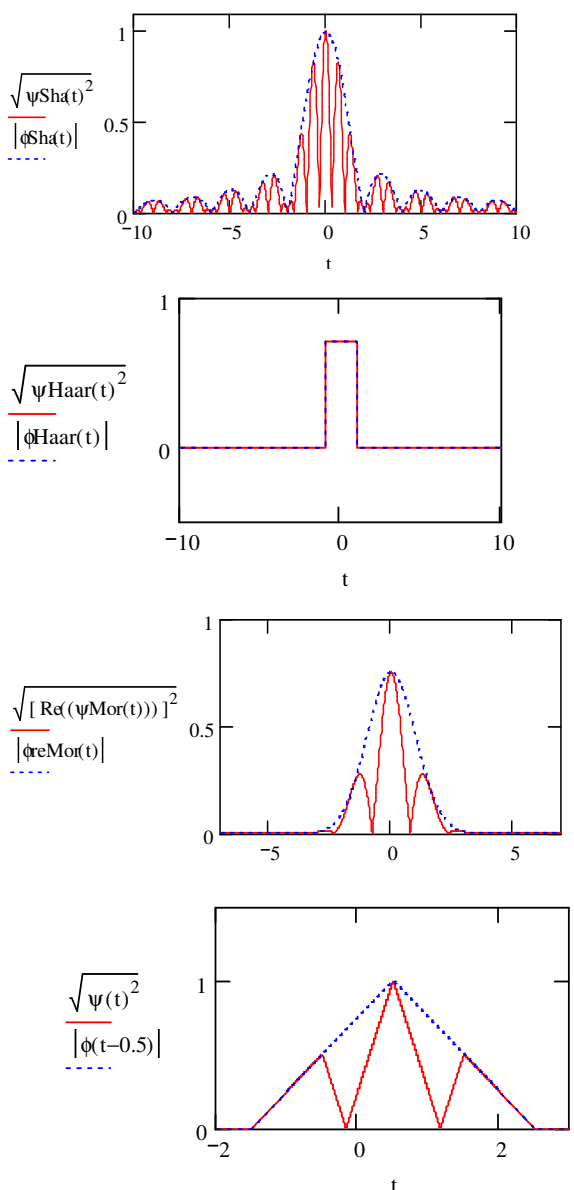

Figure 3. Illustrations of the relationship between the square root of the square wavelet function $\sqrt{\psi^{2}(t)}=|\psi(t)|$ and the modulus of the scale function $\left|\phi\left(t-t_{0}\right)\right|$ centered at $t=t_{0}$ where it reaches the maximum value. For many wavelets $\left|\phi\left(t-t_{0}\right)\right|$ is the envelope of $\sqrt{\psi^{2}(t)}$ (a) Shannon, (b) Haar (c) real Morlet (d) nonorthogonal hat wavelet.
Let $\chi(t)$ be the envelope of the wavelet modulus, $|\psi(t)|$. Since that $\lim _{|t| \rightarrow \infty} \psi(t)=0$, then $\lim _{|t| \rightarrow \infty} \gamma(t)=0$. Assuming that $\int_{-\infty}^{+\infty}|\gamma(t)| d t=E^{\prime}<+\infty$, then $\int_{-\infty}^{+\infty}|\gamma(t)| / E^{\prime} d t=1$. It is proposed to adopt $\phi(t):=\chi(t) / E^{\prime}$ as a scale function. Now, it is simple to prove that this function is orthogonal to the mother wavelet, i.e. $\langle\phi(t), \psi(t)\rangle=0$, when the wavelet is generated by a probability distribution (the blur derivative approach [12]). Denoting by $p(t)$ the probability distribution, then $p(t) \rightarrow \frac{d p}{d t}=-\psi$. In this case,

$$
<\gamma(t), \psi(t)>=\int_{-\infty}^{+\infty} p(t) \cdot \frac{-d p}{d t} d t=-\int_{-\infty}^{+\infty} \frac{1}{2} \cdot \frac{d p^{2}}{d t} d t
$$

But $\tilde{p}(t):=p^{2}(t) / \int_{-\infty}^{+\infty} p^{2}(t) d t$ is itself a probability distribution, which generates a new wavelet, say $d \tilde{p} / d t=-\tilde{\psi}$. Therefore, the orthogonally scale-wavelet follows:

$$
<\gamma(t), \psi(t)>=K \int_{-\infty}^{+\infty} \frac{d p^{2}}{d t} d t=K \int_{-\infty}^{+\infty} \widetilde{\psi}(t) d t=0 .
$$

For other wavelets (Fig. 4), it seems that there exists hitherto some kind of relationship, although being much unclear and deserves further investigation. The full-rectified scale function seems to describe a probability distribution, which generates the wavelet or is associated with. For instance, gauss1 and Mexican hat are generated from a Gaussian distribution (Fig.4b).
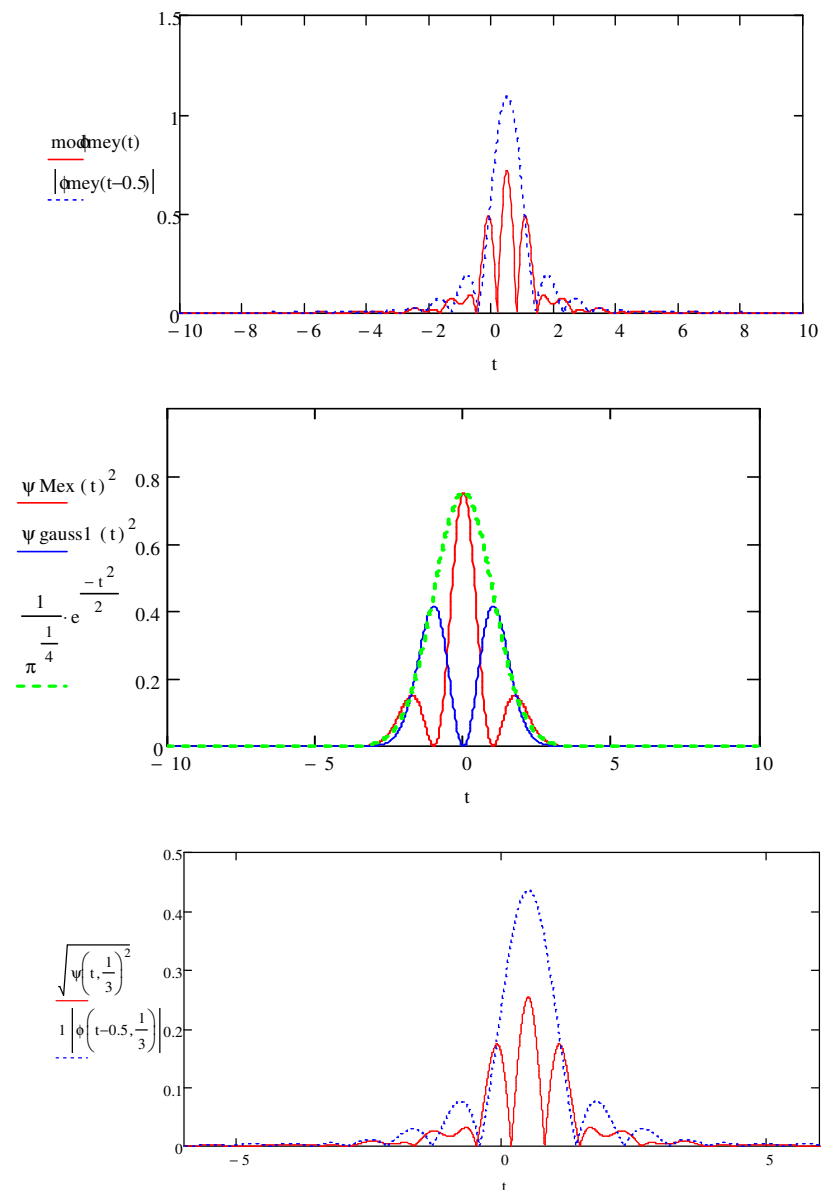

Figure 4. Illustrations of the square root of the square wavelet function $\sqrt{\psi^{2}(t)}=|\psi(t)|$ and the modulus of the scale function $\left|\phi\left(t-t_{0}\right)\right|$ centered at $t=t_{0}$ where it reaches the maximum value. (a) Meyer, (b) gauss1, Mexhat (c) deO wavelet. 


\section{CONCLUSIONS}

A novel version of the modulation theorem was presented in the framework of continuous wavelets. The requirements of bandwidth were also evaluated for a number of wavelets. Particularly, these results can be valuable for digital modulation schemes [13], multicarrier systems and OFDM wavelet-based systems [22]. Another interesting finding is concerning the (possible) relationship between the waveforms of the scale function $\phi(t)$ and the waveletderived function $\sqrt{\psi^{2}(t)}$.

\section{REFERENCES}

[1] Christopoulos, C., Skodras A., Ebrahimi, T. The JPEG2000 Still Image Coding System, IEEE Trans. On Consumer Electronics, 2000, vol.46, pp.1103-1127. doi $10.1109 / 30.920468$

[2] Farge, M. Wavelet transforms and their Application to Turbulence, Ann. Rev. Fluid Mech., 1992, vol.24, pp.395-457, doi $>10.1146$ /annurev.fl.24.010192.002143

[3] Akay, A. Wavelet Applications in Medicine, IEEE Spectrum, May, 1997, pp.50-56. doi> 10.1109/6.590747

[4] Torrence, C. and Compo, G.P. A Practical Guide to Wavelet Analysis, Bull. of the Amer. Meteorology Soc.,1998, vol.79, pp.61-78.

[5] Percival, D.B. and Walden, A.T. Wavelet Methods for Time Series Analysis, Cambridge Press (594p.),2000.

[6] de Oliveira, H.M. Análise de Sinais para Engenheiros: Uma Abordagem via Wavelets, Editora Brasport, Rio de Janeiro, 2007.

[7] de Oliveira, H.M., Falk, T.H., Távora, R.G.F. Decomposição Wavelet sobre Corpos Finitos, Rev. da Soc. Bras. de Telecomunicações, Número especial, Campinas, SP, 2002, vol. 17, n.1, pp. 38 - 47.

[8] de Oliveira, H.M., Silva, H.A.N., Bouton, E.A. Wavelet Shift-Keying: A New Digital Modulation, Anais do XX Simpósio Bras. de Telecomunicações, Rio de Janeiro, 5-8 Outubro, 2003.
[9] de Oliveira, H.M. and Bouton, E.A. Multiresolution Division Multiplex (MRDM): A New Wavelet-based Multiplex System, VI International Telecommunications Symposium (ITS2006), September 3-6, Fortaleza, Brazil. 2006. doi $>10.1109 /$ ITS.2006.4433262

[10] Lathi, B.P. and Ding, Z. Modern Digital and Analog Communication Systems, 4th Ed, Oxford University press, 2008.

[12] Mallat, S. Une Exploration des Signaux en Ondelettes, Palaiseau: Les Editions de l'École Polytechnique, 2000.

[13] Burrus, C.S., Gopinath, R.A., Guo, H. Introduction to Wavelets and the Wavelet Transform--A Primer. Prentice-Hall, Englewood Cliffs, NJ, 1998.

[14] de Oliveira, H.M., Soares, L.R., Falk, T.H. A Family of Wavelets and a New Orthogonal Multiresolutional Analysis Based on the Nyquist Criterion, IEEE SBrT Int. Telecomm. Symposium, Natal, Sept., 2002.

[15] de Oliveira, H.M. and Araujo, G.A.A. Compactly Supported Onecyclic Wavelets Derived from Beta Distributions. Journal of Communication and Information Systems, 2006, vol. 20, p. 27-33.

[16] Wozencraft J.M. and Jacobs, I.M. Principles of Communication Engineering, New York: Wiley, 1967.

[17] Slepian, D. On bandwidth, Proceedings of the IEEE, March, 1976, vol. 64 , pp. 292- 300 .

[18] Landau H.J. and Pollak, H.O. Prolate spheroidal wave functions, Fourier analysis and uncertainty: Part I, Bell System Tech. Journal, 1961, vol. 40, pp. 65-84.

[19] Gabor, D. Theory of Communications, J. IEE (Londres), 1946, vol. 93, pp. 429-457.

[20] de Oliveira, H.M. and Souza, D.F. Wavelet Analysis as an Information Processing Technique. In: VI International Telecommunications Symposium (ITS2006), 2006, Fortaleza. doi $>10.1109 /$ ITS.2006.4433232

[21] de Oliveira H.M., Lins R.D. Taylor Series as Wide-sense Biorthogonal Wavelet Decomposition. In: XXII Simpósio Brasileiro de Telecomunicações, Campinas, SP. Anais do XXII Simpósio Brasileiro de Telecomunicações. Campinas, SP, 2005, pp. 1176-1181.

[22] Akansu, A.N. and Medley, M.J. Wavelet, subband and block transforms in Communication and Multimedia, Kluwer Pub., 1999.

APPENDIX A - Close expression for time and frequency resolution of beta wavelets with parameters $\alpha$ and $\beta$.

$$
\begin{aligned}
& \bar{t}(\alpha, \beta)=\frac{1}{4} \cdot \frac{(\beta-1) \cdot \beta \cdot \Gamma(2 \alpha+1) \Gamma(2 \beta-3)}{B(\alpha, \beta)^{2} \cdot(\alpha+\beta)^{2} \cdot(\alpha+\beta+1) \cdot \Gamma(2(\alpha+\beta-2))} \\
& \Delta_{t}=\frac{\bar{t}(\alpha, \beta)\left\{\left(12 \alpha-4\left(\alpha^{2}+\alpha \beta\right)+6 \beta-9\right)+\bar{t}(\alpha, \beta) \cdot\left(2 \alpha^{2}-9 \alpha+4 \alpha \beta+9+2 \alpha \beta^{2}\right\}\right.}{(2 \alpha+2 \beta-3) \cdot(\alpha+\beta-3)} \\
& \Delta_{w}=\sqrt{\frac{3\left(\beta^{4}+\alpha^{2} \beta^{2}-3 \alpha^{2} \beta-13 \alpha \beta^{2}+2 \alpha^{2}+31 \alpha \beta+37 \beta^{2}-18 \alpha-78 \beta+40\right) \cdot \Gamma(2 \beta-5) \cdot \Gamma(2 \alpha+2 \beta-4)}{(2 \alpha-5) \cdot \Gamma(2 \alpha+2 \beta-6) \cdot \Gamma(2 \beta-3) \cdot\left(\beta^{2}+\alpha \beta-\alpha-4 \beta-3\right)}}
\end{aligned}
$$

The figure in this appendix shows the tradeoff between time and frequency resolution for the $(\alpha, 3)$ beta wavelet.
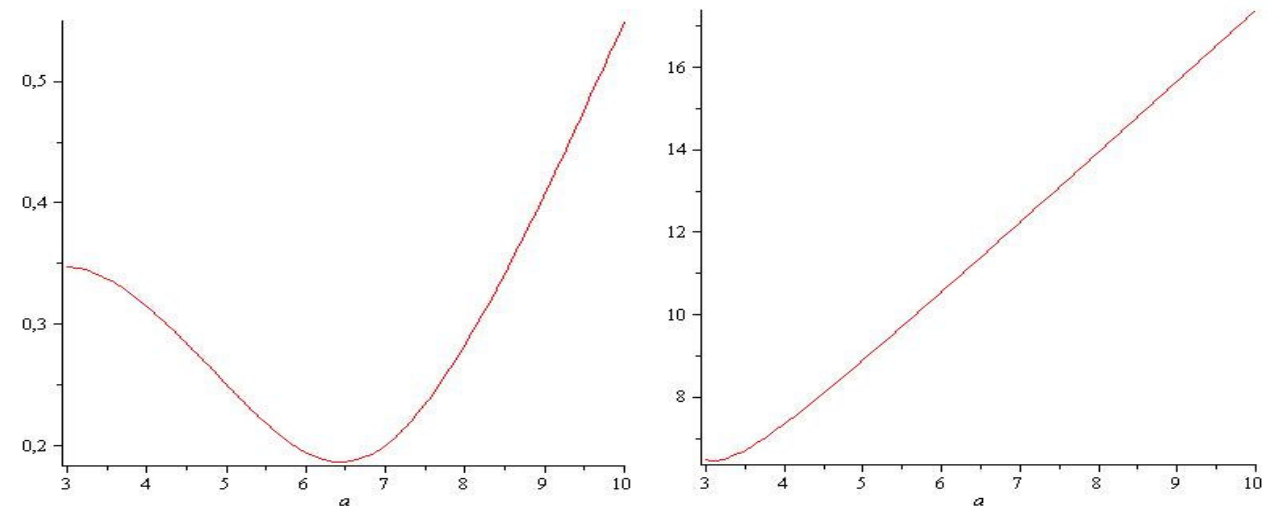

Figure 1A. Variation of the resolution of the beta wavelet: (a) time resolution, (b) frequency resolution. The abscissa shows the variation of the parameter $\alpha$ while keeping $\beta=3$ constant. To be remarked that $\alpha=6 \beta=3$ achieve better joint resolution. Similar behavior was obtained for others beta wavelets. 
XXVII SIMPÓSIO BRASILEIRO DE TELECOMUNICAÇÕES - SBrT 2009, DE 29 DE SETEMBRO A 2 DE OUTUBRO DE 2009, BLUMENAU, SC

APPENDIX B - Waveforms of a few known continuous wavelets in both time and frequency domain.

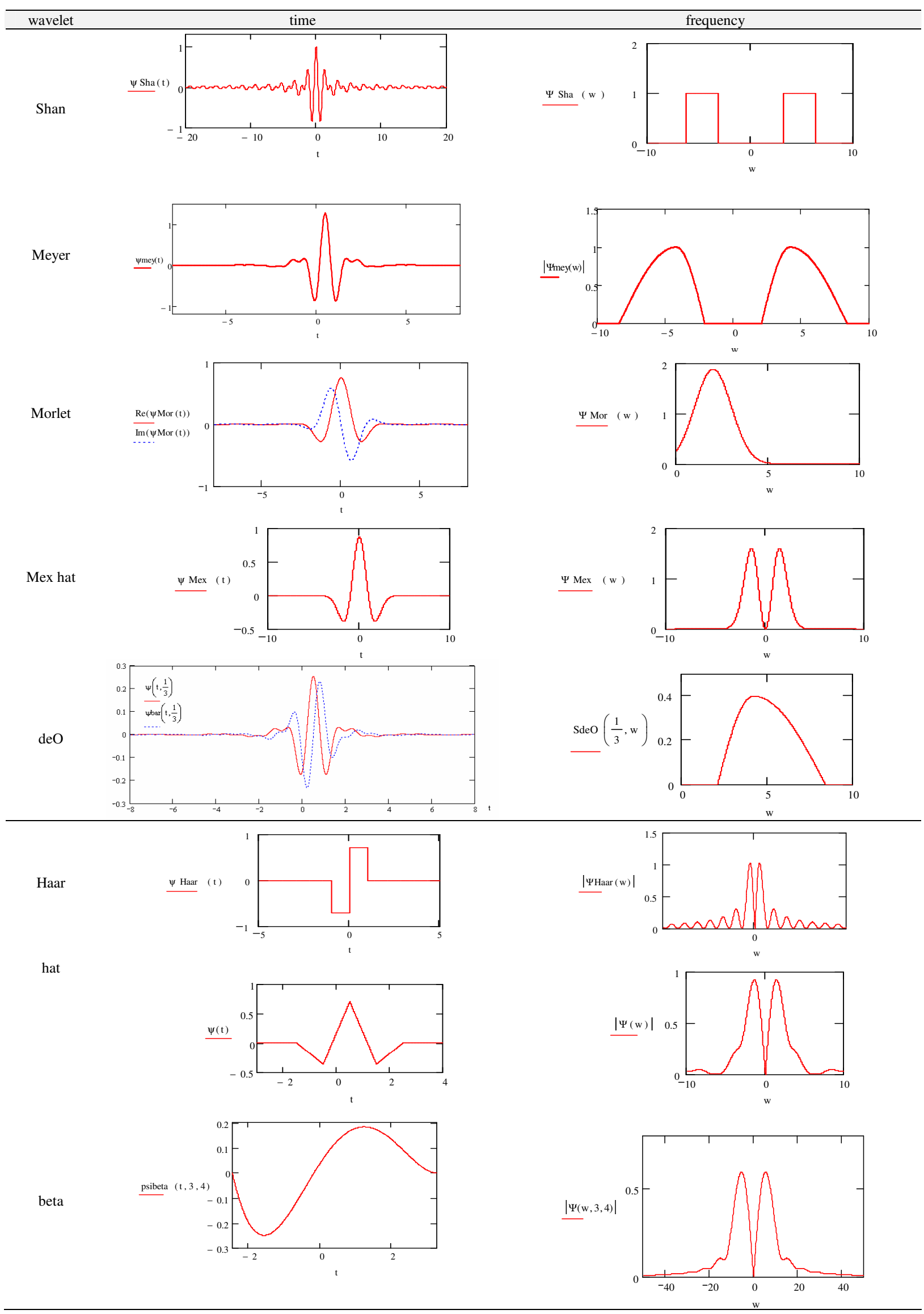

